\title{
The Perfect Binary One-Error-Correcting Codes of Length 15: Part I-Classification
}

\author{
Patric R. J. Östergård, Olli Pottonen
}

\begin{abstract}
A complete classification of the perfect binary one-error-correcting codes of length 15 as well as their extensions of length 16 is presented. There are 5983 such inequivalent perfect codes and 2165 extended perfect codes. Efficient generation of these codes relies on the recent classification of Steiner quadruple systems of order 16 . Utilizing a result of Blackmore, the optimal binary one-error-correcting codes of length 14 and the $(15,1024,4)$ codes are also classified; there are 38408 and 5983 such codes, respectively.

\section{Index Terms}

classification, Hamming code, perfect binary code, Steiner system
\end{abstract}

\section{INTRODUCTION}

Consider the space $\mathbb{F}_{2}^{n}$ of dimension $n$ over the Galois field $\mathbb{F}_{2}=\{0,1\}$. A binary code of length $n$ is a subset of $\mathbb{F}_{2}^{n}$. The (Hamming) distance $d(\mathbf{x}, \mathbf{y})$ between two codewords $\mathbf{x}, \mathbf{y}$ is the number of coordinates in which they differ, and the (Hamming) weight $\mathrm{wt}(\mathbf{x})$ is the number of nonzero coordinates. The support of a codeword is the set of nonzero coordinates, $\operatorname{supp}(\mathbf{x})=\left\{i: x_{i} \neq 0\right\}$. Accordingly, $d(\mathbf{x}, \mathbf{y})=\operatorname{wt}(\mathbf{x}-\mathbf{y})=|\operatorname{supp}(\mathbf{x}-\mathbf{y})|$.

A code has minimum distance $d$ if $d$ is the largest integer such that the distance between any distinct codewords is at least $d$. Then the balls of radius $\lfloor(d-1) / 2\rfloor$ centered around the codewords are nonintersecting, and the code is said to be a $\lfloor(d-1) / 2\rfloor$-error-correcting code. If these balls tile the whole space, then the code is called perfect. The parameters of perfect codes over an alphabet of prime order are well known [1], and perfect binary codes exist with $d=1 ; d=n ; d=(n-1) / 2$ for odd $n$; $d=3, n=2^{m}-1$ for $m \geq 2$; and $d=7, n=23$. The first three types of codes are called trivial, the fourth has the parameters of Hamming codes, and the last one is the binary Golay code. A perfect code with minimum distance $d$ is also called a $\lfloor(d-1) / 2\rfloor$-perfect code.

A binary code with length $n$, minimum distance $d$, and $M$ codewords is called a $(n, M, d)$ code. In this notation a binary 1-perfect code is a $\left(2^{m}-1,2^{2^{m}-m-1}, 3\right)$ code. Two related families are the extended and shortened 1-perfect codes, which have parameters $\left(2^{m}, 2^{2^{m}-m-1}, 4\right)$ and $\left(2^{m}-2,2^{2^{m}-m-2}, 3\right)$, respectively.

Existence of binary 1-perfect codes follows from the existence of Hamming codes, which are the unique linear 1-perfect codes. Still constructing all 1-perfect codes is a longstanding open problem. It makes sense to approach this issue by considering the number of inequivalent codes (or more formally the number of equivalence classes). Two codes are said to be equivalent if one is obtained from the other by permuting coordinates and adding a constant vector; a formal definition appears in Section II.

There is trivially a unique 1-perfect code of length 3. Zaremba [2] showed that also the 1-perfect code of length 7 is unique. However, already the next case of length 15 has until this work withstood all attempts of complete classification, although several constructions of such codes have been published; see the surveys [3], [4]. It turns out that these results were not far from a complete classification as for the number of codes found. The growth of the number of 1-perfect binary codes is double exponential in the

This work was supported in part by the Graduate School in Electronics, Telecommunication and Automation and by the Academy of Finland, Grant Numbers 107493 and 110196.

P. R. J. Östergård and O. Pottonen are with the Department of Communications and Networking, Helsinki University of Technology TKK, P.O.Box 3000, FI-02015 TKK, Finland (e-mail: patric.ostergard@tkk.fi, olli.pottonen@tkk.fi) 
length of the code, see [5] for a lower bound on this number. For an in-depth treatment of the topic of classifying combinatorial objects, see [6].

The aim of the current work is to obtain a complete classification of inequivalent 1-perfect binary codes of length 15. By computer search it is here shown that their number is 5983 . Also the codes obtained by extending, shortening or extending and shortening are classified; the numbers of $(16,2048,4)$, $(14,1024,3)$ and $(15,1024,4)$ codes turn out to be 2165,38408 and 5983 respectively.

In the rest of the paper we document the classification of the extended 1-perfect codes of length 16, which yields classifications of the 1-perfect codes of length 15 and the shortened 1-perfect codes of length 14. In Section III we define some concepts and consider construction of extended 1-perfect codes via Steiner systems. In Section III we present algorithms for detecting and rejecting equivalent codes, and in Section IV], we take a brief look at the results; a separate, more detailed study of the classified codes will appear in a separate paper [7]. Finally, in Section $\nabla$ we give a consistency check for gaining confidence in the computational results.

\section{PReliminaries And CONSTRUCTION}

A permutation $\pi$ of the set $\{1,2, \ldots, n\}$ acts on codewords by permuting the coordinates: $\pi\left(\left(c_{1}, c_{2}, \ldots, c_{n}\right)\right)=$ $\left(c_{\pi^{-1}(1)}, c_{\pi^{-1}(2)}, \ldots, c_{\pi^{-1}(n)}\right)$. Pairs $(\pi, \mathbf{x})$ form the wreath product $S_{2} 2 S_{n}$, which acts on codes as $(\pi, \mathbf{x})(C)=$ $\pi(C+\mathbf{x})=\pi(C)+\pi(\mathbf{x})$. Two codes, $C_{1}$ and $C_{2}$, are isomorphic if $C_{1}=\pi\left(C_{2}\right)$ for some $\pi$ and equivalent if $C_{1}=\pi\left(C_{2}+\mathbf{x}\right)$ for some $\pi, \mathbf{x}$.

The automorphism group of a code $C$, Aut $(C)$, is the group of all pairs $(\pi, \mathbf{x})$ such that $C=\pi(C+\mathbf{x})$. Two important subgroups of $\operatorname{Aut}(C)$ are the group of symmetries,

$$
\operatorname{Sym}(C)=\{\pi: \pi(C)=C\}
$$

and the kernel

$$
\operatorname{Ker}(C)=\{\mathbf{x}: C+\mathbf{x}=C\} .
$$

If the code contains the all-zero word, $\mathbf{0}$, then the elements of the kernel are codewords.

A Steiner system $S(t, k, v)$ can be viewed as a code $S \subset \mathbb{F}_{2}^{v}$ with the property that each codeword of $S$ has weight $k$, and for any $\mathbf{y} \in \mathbb{F}_{2}^{v}$ with $\operatorname{wt}(\mathbf{y})=t$, there is a unique $\mathbf{x} \in S$ such that $\operatorname{supp}(\mathbf{y}) \subseteq \operatorname{supp}(\mathbf{x})$. Usually Steiner systems are defined as set systems rather than codes, but our definition is more directly applicable for this work. The parameter $v$ is the order of the system. Steiner systems $S(2,3, v)$ and $S(3,4, v)$, which are called Steiner triple systems and Steiner quadruple systems, respectively, are related to 1-perfect codes in the following way. If $C$ is a 1-perfect binary code of length $v$ and $\mathbf{x} \in C$, then the codewords of $C+\mathbf{x}$ with weight 3 form a Steiner triple system of order $v$. Similarly, if $C$ is an extended 1-perfect binary code and $\mathrm{x} \in C$, then the codewords of $C+\mathrm{x}$ with weight 4 form a Steiner quadruple system. These systems are, respectively, the neighborhood triple system and neighborhood quadruple system associated with the code and the codeword.

As a starting point for the classification of the extended 1-perfect binary codes of length 16, we have the classification [8] of Steiner quadruple systems of order 16; there are 1054163 such designs. We want to find, for each $S(3,4,16)$, all extended 1-perfect binary codes in which it occurs. This can be done by puncturing any coordinate, augmenting the resulting code to 1-perfect codes in all possible ways, and finally extending every resulting code with a parity bit.

When augmenting a set of codewords to a 1-perfect code, we consider a 1-perfect code as a set of balls with radius one that form a partition of the ambient space. Accordingly, finding a code (with specified codewords) is a special case of the exact cover problem, where we are given a set $S$ and a collection $U$ of its subsets, and the task is to form a partition of $S$ by using sets in $U$. Let the set $Q$ contain the codewords obtained by puncturing the all-zero codeword and its neighborhood quadruple system. In this case we have $S=\mathbb{F}_{2}^{15} \backslash B(Q)$, and $U=\{B(\mathbf{x}): B(\mathbf{x}) \cap B(Q)=\emptyset\}$, where $B(\mathbf{x})=\{\mathbf{y}: d(\mathbf{x}, \mathbf{y}) \leq 1\}$ and $B(C)=\{B(\mathbf{x}): \mathbf{x} \in C\}$. We use the libexact software [9] for solving such instances of the exact cover problems. In the search we could in fact have made use of the fact that all 1-perfect binary codes 
are self-complementary-in other words, the all-one word is always in the kernel-but this would not have had any practical significance as the search was rather fast.

Since the all-zero word and its neighborhood quadruple system contain 141 of the 2048 codewords, 1907 new codewords are needed. Searching for these was a remarkably easy computational task; on average the search trees in which codes were found had 1978 nodes and those in which no codes were found had 3 nodes.

\section{ISOMORPH REJECTION}

The general framework by McKay [10] was used to carry out isomorph rejection, although a less sophisticated method would have sufficed in this work.

Recall that we augment a Steiner quadruple system $Q$ to an extended 1-perfect code $C$. We accept $C$ if it passes the following two tests; otherwise it is rejected. First we require that $C$ shall be the minimum (with respect to some practically computable total order of codes) under the action of Aut $(Q)$. Second, we compute the canonical equivalence class representative $c_{E}(C)$, consider $\pi$, $\mathbf{x}$ for which $\pi(C+\mathbf{x})=c_{E}(C)$ and require that $\mathbf{x}$ and $\mathbf{0}$ are on the same $\operatorname{Aut}(C)$ orbit (we define $c_{E}$ so that $\mathbf{x} \in C$ always holds).

When the extended 1-perfect codes have been classified, classifying the 1-perfect codes is straightforward. All 1-perfect codes are obtained by puncturing the extended codes, and the resulting 1-perfect codes are equivalent if and only if they are obtained by puncturing the same extended code at coordinates which are in the same orbit of the automorphism group.

A complete classification of the $(14,1024,3)$ codes is obtained similarly, since each such code is obtained by shortening a unique (up to equivalence) 1-perfect code of length 15; this result was proved by Blackmore [11]. Although a code can be shortened at any coordinate in two ways, by selecting the codewords with 0 or 1 in a certain coordinate, both selections lead to equivalent codes. This follows from the fact that every 1-perfect binary code is self-complementary.

Furthermore we note that any $(15,1024,4)$ code is obtained by extending a $(14,1024,3)$ code with a parity bit. Hence all such codes are obtained by shortening and extending a perfect code, or equivalently removing all words of chosen parity. As the perfect codes are self-complementary, we get (up to equivalence) same code by chosing either odd or even parity. As this mapping is reversible, we conclude that there is one-to-one correspondence between equivalence classes of $(15,2048,3)$ codes and equivalence classes of $(15,1024,4)$ codes, and in both cases their number is 5983 .

Let $C$ be a $(15,1024,4)$ code $C$ and let $C^{\prime}$ be the corresponding perfect code. The group $\operatorname{Aut}\left(C^{\prime}\right)$ contains $\operatorname{Aut}(C)$ as a subgroup, and $\operatorname{Aut}\left(C^{\prime}\right)$ has one more generator than $\operatorname{Aut}(C)$, namely the all-one codeword. Accordingly $\left|\operatorname{Aut}\left(C^{\prime}\right)\right|=2|\operatorname{Aut}(C)|$.

We still have to describe an algorithm for canonical labeling. The most straightforward approach of using the general purpose isomorphism nauty [12] is rather slow on codes as large and regular as the $(16,2048,4)$ codes; this was also noted by Phelps [13]. Hence a tailored approach is necessary. The method presented below has a lot in common with the one described in [13]. An alternative method based on minimum distance graphs would also work [14], cf. [15].

A triangle consists of 3 codewords with mutual distance 4 . Triangles constitute an easily computable and rather sensitive invariant of Steiner quadruple systems. Distinguishing the isomorphism classes of the neighborhood quadruple systems of a code also constitutes an invariant of the extended 1-perfect codes. These two invariants turned out to be useful for speeding up our computations.

A canonical isomorphism class representative $c_{I}(C)$ for a code $C$ can be computed by using nauty to label a corresponding graph canonically. Moreover, nauty computes generators of the group $\operatorname{Sym}(C)$. Canonical equivalence class representative can be defined as $c_{E}(C)=\min \left\{c_{I}(C+\mathbf{x}): \mathbf{x} \in C\right\}$, where the minimum is again taken with respect to some practically computable total order of codes.

Note that two codewords, $\mathbf{x}$ and $\mathbf{y}$, are in the same orbit of $\operatorname{Aut}(C)$ if and only if $c_{I}(C+\mathbf{x})=c_{I}(C+\mathbf{y})$. Because of this, if we know that $\mathbf{x}$ and $\mathbf{y}$ are in the same orbit, and $c_{I}(C+\mathbf{x})$ has been computed, then there is no need to compute $c_{I}(C+\mathbf{y})$. Also if $c_{I}(C+\mathbf{x})=c_{I}(C)$, then nauty yields a permutation $\pi$ 
such that $\pi(C+\mathbf{x})=C$. The pairs $(\pi, \mathbf{x})$ are coset representatives of $\operatorname{Aut}(C)$ with respect to $\operatorname{Sym}(C)$, so we get generators of the group $\operatorname{Aut}(C)$.

\section{RESULTS}

There are exactly 2165 inequivalent extended 1-perfect codes of length 16, 5983 inequivalent 1-perfect codes of length 15, 38408 shortened 1-perfect codes of length 14 and $5983(15,1024,4)$ codes. The orders of the automorphism groups of the codes are presented in Tables I, II and III. As noted in Section III, the order of the automorphism group of a $(15,1024,4)$ code is half of the order of the automorphism group of the corresponding perfect code.

The codes have been made available in electronic form by including them in the source of the arXiv version of this paper. Downloading http://arxiv.org/e-print/0806.2513v3 and uncompressing it with gunzip and tar yields the files perfect 15 and extended 16.

Only 15590 of the 1054163 nonisomorphic $S(3,4,16)$ can be augmented to a 1-perfect code, and the total number of extensions is 22814 . The computationally intensive part of this result was the earlier classification of $S(3,4,16)$, which required several years of CPU time, while all searches described in this paper took only a couple of hours of CPU time.

A detailed study of the properties of the classified codes will appear in a second part of this article [7].

TABLE I

AUTOMORPHISM GROUPS OF $(16,2048,4)$ CODES

\begin{tabular}{rrrrrr}
\hline $\mid$ Aut $(C) \mid$ & $\#$ & $\mid$ Aut $(C) \mid$ & $\#$ & $\mid$ Aut $(C) \mid$ & $\#$ \\
\hline 128 & 11 & 5376 & 1 & 196608 & 6 \\
192 & 5 & 6144 & 23 & 262144 & 3 \\
256 & 105 & 8192 & 174 & 344064 & 1 \\
384 & 9 & 10752 & 2 & 393216 & 3 \\
512 & 377 & 12288 & 22 & 524288 & 2 \\
672 & 2 & 16384 & 103 & 688128 & 1 \\
768 & 19 & 24576 & 12 & 786432 & 2 \\
1024 & 416 & 32768 & 47 & 1572864 & 3 \\
1344 & 1 & 43008 & 2 & 2359296 & 1 \\
1536 & 21 & 49152 & 18 & 2752512 & 1 \\
1920 & 1 & 61440 & 1 & 3145728 & 1 \\
2048 & 394 & 65536 & 33 & 5505024 & 2 \\
2688 & 1 & 86016 & 3 & 6291456 & 1 \\
3072 & 18 & 98304 & 12 & 660602880 & 1 \\
4096 & 298 & 131072 & 6 & & \\
\hline
\end{tabular}

\section{CONSISTENCY CHECK}

To get confidence in the results, we performed a consistency check similar to the one used, for example, in [8]. In this check we count the total number of codes in two different ways and ensure that the results agree.

First we consider the set $\mathcal{C}$ of equivalence class representatives obtained in the classification. By the orbit-stabilizer theorem, the total number of extended 1-perfect codes is

$$
\sum_{C \in \mathcal{C}} \frac{16 ! \cdot 2^{16}}{\operatorname{Aut}(C)}
$$

where $16 ! \cdot 2^{16}$ is the order of the wreath product group acting on the codes.

Let $\mathcal{Q}$ consist of the representative Steiner quadruple systems, and let $E(Q)$ be the number of all extended 1-perfect codes obtained by augmenting $Q$. Applying the orbit-stabilizer theorem, we get the expression

$$
\frac{1}{2048} \sum_{Q \in \mathcal{Q}} \frac{16 ! \cdot 2^{16} \cdot E(Q)}{\operatorname{Aut}(Q)}
$$


TABLE II

AUTOMORPHISM GROUPS OF $(15,2048,3)$ CODES

\begin{tabular}{rrrrrr}
\hline $\mid$ Aut $(C) \mid$ & $\#$ & $\mid$ Aut $(C) \mid$ & $\#$ & $\mid$ Aut $(C) \mid$ & $\#$ \\
\hline 8 & 3 & 512 & 1017 & 24576 & 7 \\
12 & 3 & 672 & 3 & 32768 & 8 \\
16 & 5 & 768 & 32 & 43008 & 4 \\
24 & 10 & 1024 & 697 & 49152 & 10 \\
32 & 138 & 1536 & 17 & 65536 & 5 \\
42 & 2 & 2048 & 406 & 98304 & 1 \\
48 & 12 & 2688 & 1 & 131072 & 1 \\
64 & 542 & 3072 & 37 & 172032 & 1 \\
96 & 22 & 3840 & 1 & 196608 & 5 \\
120 & 1 & 4096 & 202 & 344064 & 2 \\
128 & 1230 & 5376 & 4 & 393216 & 2 \\
192 & 18 & 6144 & 35 & 589824 & 1 \\
256 & 1319 & 8192 & 94 & 41287680 & 1 \\
336 & 3 & 12288 & 7 & & \\
384 & 30 & 16384 & 44 & & \\
\hline
\end{tabular}

TABLE III

AUTOMORPHISM GROUPS OF $(14,1024,3)$ CODES

\begin{tabular}{rrrrrr}
\hline $\mid$ Aut $(C) \mid$ & $\#$ & $\mid$ Aut $(C) \mid$ & $\#$ & $\mid$ Aut $(C) \mid$ & $\#$ \\
\hline 1 & 5 & 168 & 1 & 8192 & 80 \\
2 & 75 & 192 & 80 & 12288 & 18 \\
3 & 8 & 256 & 4392 & 16384 & 14 \\
4 & 425 & 336 & 5 & 21504 & 1 \\
6 & 39 & 384 & 114 & 24576 & 15 \\
8 & 1162 & 512 & 2469 & 32768 & 14 \\
12 & 56 & 768 & 30 & 49152 & 1 \\
16 & 3465 & 1024 & 1346 & 65536 & 1 \\
21 & 4 & 1344 & 1 & 86016 & 1 \\
24 & 39 & 1536 & 54 & 98304 & 2 \\
32 & 7311 & 2048 & 527 & 172032 & 1 \\
48 & 59 & 2688 & 6 & 196608 & 2 \\
64 & 9068 & 3072 & 55 & 1376256 & 1 \\
96 & 49 & 4096 & 222 & & \\
128 & 7172 & 6144 & 18 & & \\
\hline
\end{tabular}

where the division by 2048 is necessary since each code is counted once for each codeword. Both formulas yield the same result, 2795493027033907 200. Similarly we also counted the 1-perfect codes and shortened 1-perfect codes in two different ways; their number is 1397746513516953600 . Indeed, there are twice as many extended 1-perfect codes as there are 1-perfect codes, since each 1-perfect code admits two extensions: one with even parity bit and one with odd. Similarly, we get a bijection from the 1-perfect codes to shortened 1-perfect codes if we shorten each code by taking, for instance, the codewords with value 0 in coordinate 15 and removing that coordinate. Thus there are equally many 1-perfect codes and shortened 1-perfect codes.

\section{REFERENCES}

[1] F. J. MacWilliams and N. J. A. Sloane, The Theory of Error-Correcting Codes. Amsterdam: North-Holland, 1977.

[2] S. K. Zaremba, "Covering problems concerning Abelian groups," J. London Math. Soc., vol. 27, pp. 242-246, 1952.

[3] T. Etzion and A. Vardy, "Perfect binary codes: constructions, properties, and enumeration," IEEE Trans. Inform. Theory, vol. 40, pp. 754-763, 1994.

[4] O. Heden, "A survey of perfect codes," Adv. Math. Commun., vol. 2, pp. 223-247, 2008.

[5] D. S. Krotov and S. V. Avgustinovich, "On the number of 1-perfect binary codes: a lower bound," IEEE Trans. Inform. Theory, vol. 54, pp. 1760-1765, 2008.

[6] P. Kaski and P. R. J. Östergård, Classification Algorithms for Codes and Designs. Berlin: Springer, 2006.

[7] P. R. J. Östergård, O. Pottonen and K. T. Phelps, "The Perfect Binary One-Error-Correcting codes of Length 15: Part II-Properties", submitted for publication. Preprint at arXiv:0903:2749. 
[8] P. Kaski, P. R. J. Östergård, and O. Pottonen, "The Steiner quadruple systems of order 16,” J. Combin. Theory Ser. A, vol. 113, pp. 1764-1770, 2006.

[9] P. Kaski and O. Pottonen, "libexact user's guide, version 1.0.", Helsinki Institute for Information Technology HIIT, Helsinki, Tech. Rep. 2008-1, 2008.

[10] B. D. McKay, "Isomorph-free exhaustive generation," J. Algorithms, vol. 26, pp. 306-324, 1998.

[11] T. Blackmore, "Every binary $\left(2^{m}-2,2^{2^{m}-2-m}, 3\right)$ code can be lengthened to form a perfect code of length $2^{m}-1$," IEEE Trans. Inform. Theory, vol. 45, pp. 698-700, 1999.

[12] B. D. McKay, "nauty user's guide (version 1.5)," Computer Science Department, Australian National University, Canberra, Tech. Rep. TR-CS-90-02, 1990.

[13] K. T. Phelps, “An enumeration of 1-perfect binary codes,” Australas. J. Combin., vol. 21, pp. 287-298, 2000.

[14] I. Yu. Mogilnykh, P. R. J. Östergård, O. Pottonen and F. I. Solov’eva, "Reconstructing Extended Perfect Binary One-Error-Correcting Codes from Their Minimum Distance Graphs”, IEEE Trans. Inform. Theory, vol. 55, pp. 2622-2625, 2009.

[15] K. Phelps and M. Levan, "Switching equivalence classes of perfect codes", Des. Codes Cryptogr. vol. 16, pp. 179-184, 1999. 